\title{
Papillary Urothelial Neoplasm of Low Malignant Potential: A Case Report
}

\author{
Nanda Patil ${ }^{1}$, Avinash Mane $^{2}$, Jyoti Tele ${ }^{3}$, Pranita Warhate ${ }^{3}$ \\ ${ }^{1}$ Professor, Department of Pathology, Krishna Institute of Medical Science, Karad, India \\ ${ }^{2}$ Assistant Professor, Department of Pathology, Krishna Institute of Medical Science, Karad, India \\ ${ }^{3,4}$ Tutor, Department of Pathology, Krishna Institute of Medical Science, Karad, India
}

\begin{abstract}
Papillary urothelial neoplasm of low malignant potential (PUNLMP) is a new histological diagnosis defined in the WHO 1998 and revised in 2004 which is used for urothelial neoplasm showing multilayering of epithelium with minimal or absent cytological atypia. The neoplasm has low risk of progression and is seen in adults older than 20 years. We report a case of PUNLMP in a 20 year old female patient.
\end{abstract}

Keywords: Bladder, Urothelial Neoplasm, PUNLMP

\section{Introduction}

Papillary urothelial neoplasm of low malignant potential (PUNLMP) is a new histological diagnosis for tumor of bladder defined by WHO in 1998 and revised in year 2004 .

The term PUNLMP is used for urothelial neoplasm of bladder which isnon invasive, showing multilayering of epithelium with minimal or absent cytological atypia. PUNLMP is a neoplasm with a low risk of progression. ${ }^{[1]}$ These neoplasms are usually seen in the patients younger than 18 years of age. ${ }^{[2]} \mathrm{We}$ report a case of bladder PUNLMP in a 20 year old female patient.

\section{Case Report}

A 20 year old female patient presented with painless intermittent hematuria. An abdominal ultrasound revealed small isoechoicpolypoidal lesion along right posterolateral wall of urinary bladder measuring $12 \times 4 \mathrm{~mm}$ with a narrow base. After surgical resection the tumor was sent for histological examination.

Pathological examination revealed anurothelial neoplasm with delicate and discrete papillae having thin fibrovascular core. The papillae were lined by urothelium showing multilayering of cells with normal polarity. The nuclei were uniformly and evenly distributed and revealed mild enlargement but no atypia. Mitosis was absent (Fig 1, 2). Considering these features, the lesion was diagnosed as PUNLMP and the patient was advised close follow up with ultrasound and urine analysis.

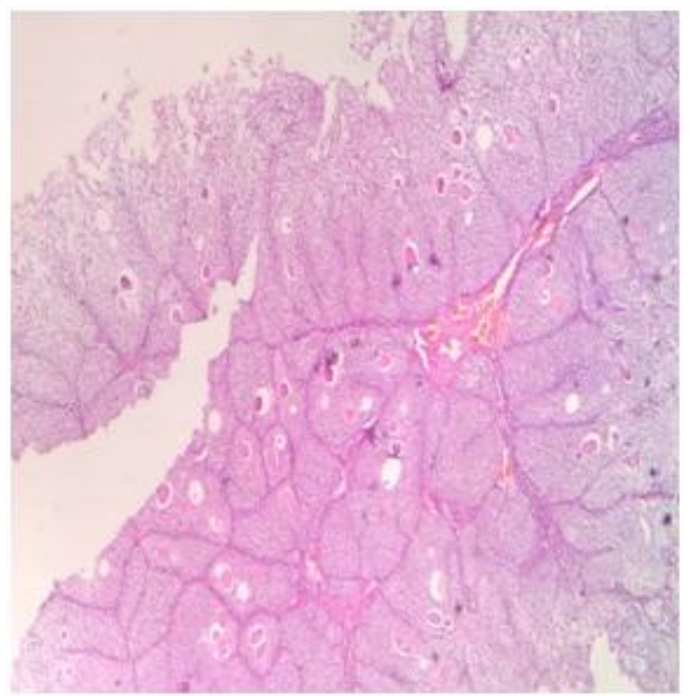

Figure 1: Microscopy reveals delicate discrete papillae with thin fibrovascular core (40X, H\&E)

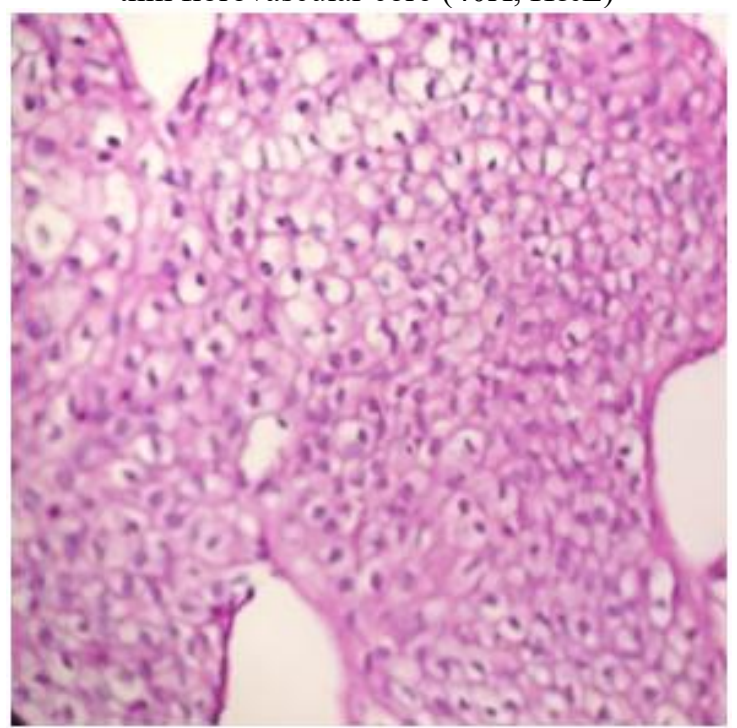

Figure 2: Papillae showing multilayered urothelium with normal polarity \& without atypia (400X, H\&E) 


\section{International Journal of Science and Research (IJSR) \\ ISSN (Online): 2319-7064}

Index Copernicus Value (2015): 78.96 | Impact Factor (2015): 6.391

\section{Discussion}

PUNLMP is a term revised by WHO for neoplasm of bladder which reveal multilayering of urothelium with minimal or absent cytological atypia. These neoplasms are seen in a younger age group as seen in our case who presented at the age of 20 years with complaints of hematuria as described by various authors. ${ }^{[2]}$ As these patients present in younger age group the diagnosis can be missed clinically because of low index of suspicion.

Ultrasound examination helps to detect these neoplasms as cystoscopy is usually not preferred in this age group due to risk of urethral manipulation. ${ }^{[3]}$ In our case, the lesion was detected with ultrasound examination and the diagnosis was given by histopathological examination. Possible genetic link for PUNLMP in young cases has been observed but overlapping with environmental risk factors makes it difficult to provide a definitive conclusion. ${ }^{[4]}$

PUNLMPs have variable and increased incidence of rate of recurrence $(10-45 \%) \&$ progression $(0-29 \%) .{ }^{[5,}{ }^{6]}$ These patients should be monitored regularly for recurrence or progression. Invasive cystoscopy is usually not recommended due to risk of urethral damage and need of anesthesia, Noninvasive modalities like ultrasound, urine analysis and cytology are recommended for regular follow up. Immunohistochemical markers like negativeP53and normal CK2 are helpful in predicting recurrence and progression of these tumors. ${ }^{[7]}$ Our case is on regular follow up and the outcome is uneventful.

\section{Conclusion}

Papillary urothelial neoplasm of low malignant potential is a new histological term defined by WHO for tumors of bladder which reveal multilayering of epithelium with minimal or absent cytological atypia. These tumors have excellent long term prognosis and low rate of recurrence.

The diagnosis is missed clinically as these patients present in younger age group. High clinical suspicion should be kept for these cases. Minimally invasive diagnostic modalities like ultrasonography and urine analysis should be used for follow up.

\section{References}

[1] Fine SW, Humphrey PA, Dehner LP, Amin MB, Epstein JI. Urothelial neoplasms in patients 20 years or younger: a clinicopathological analysis using the world health organization 2004 bladder consensus classification. J Urol 2005;174:1976- 80.

[2] Alanee S, Shukla AR. Bladder malignancies in children aged $<18$ years: results from the surveillance, epidemiology and end results database. BJU Int 2010;106:557-60.

[3] L. Gao, D.X. Tang, M.J. LI, S. XU, Papillary urothelial neoplasm of low malignant potential in a 9-year-old boy: A case report, Hong Kong J. Paediatr. (new series)2014;19:262-264
[4] Mueller CM, Caporaso N, Greene MH. Familial and genetic riskof transitional cell carcinoma of the urinary tract. UrolOncol2008;26:451-64.

[5] Jones TD, Cheng L. Papillary urothelial neoplasm of lowmalignant potential: evolving terminology and concepts. J Urol2006;175:1995-2003.

[6] Lee TK, Chaux A, Karram S, et al. Papillary urothelial neoplasm of low malignant potential of the urinary bladder:clinicopathologic and outcome analysis from a single academiccenter. Hum Pathol 2011;42:1799-803.

[7] Yin H, Leong AS. Histologic grading of noninvasive papillaryurothelial tumors: validation of the 1998 WHO/ISUP system byimmunophenotyping and followup. Am J Clin Pathol 2004;121:679-87. 